\title{
Epidemiology and burden of alopecia areata: a systematic review
}

This article was published in the following Dove Press journal:

Clinical, Cosmetic and Investigational Dermatology

24 July 2015

Number of times this article has been viewed

\section{Alexandra CVillasante Fricke Mariya Miteva \\ Department of Dermatology and Cutaneous Surgery, University of Miami Miller School of Medicine, Miami, FL, USA}

Background: Alopecia areata (AA) is an autoimmune disorder characterized by patches of non-scarring alopecia affecting scalp and body hair that can be psychologically devastating. AA is clinically heterogenous, and its natural history is unpredictable. There is no preventative therapy or cure.

Objective: The objective of this study is to provide an evidence-based systematic review on the epidemiology and the burden of AA.

Methods and selection criteria: A search was conducted of the published, peer-reviewed literature via PubMed, Embase, and Web of Science. Studies published in English within the last 51 years that measured AA's incidence, prevalence, distribution, disability-adjusted life years (DALYs), quality of life, and associated psychiatric and medical comorbidities were included. Two authors assessed studies and extracted the data.

Results: The lifetime incidence of AA is approximately $2 \%$ worldwide. Both formal population studies found no sex predominance. First onset is most common in the third and fourth decades of life but may occur at any age. An earlier age of first onset corresponds with an increased lifetime risk of extensive disease. Global DALYs for AA were calculated at 1,332,800 in 2010. AA patients are at risk for depression and anxiety, atopy, vitiligo, thyroid disease, and other autoimmune conditions.

Conclusion: AA is the most prevalent autoimmune disorder and the second most prevalent hair loss disorder after androgenetic alopecia, and the lifetime risk in the global population is approximately $2 \%$. AA is associated with psychiatric and medical comorbidities including depression, anxiety, and several autoimmune disorders, and an increased global burden of disease.

Keywords: hair loss, hair, prevalence, incidence, burden of disease

\section{Introduction}

Alopecia areata (AA) is a common, clinically heterogenous, immune-mediated, nonscarring hair loss disorder. ${ }^{1-3}$ The disease may be limited to one or more discrete, wellcircumscribed round or oval patches of hair loss on the scalp or body, or it may affect the entire scalp (alopecia totalis) or the entire body (alopecia universalis). ${ }^{1,2}$ Moreover, the course of disease is unpredictable, with spontaneous regrowth of hair occurring in $80 \%$ of patients within the first year, and sudden relapse at any given time. ${ }^{2,3}$ In AA, CD4+ and CD8+ T-cells violate the immune privilege of the anagen hair follicle, leading to loss of the growing hair shaft. ${ }^{1,3,4} \mathrm{CD} 8+$ T-cells are present in significantly greater quantities than CD4+ cells, and a subset of them known as CD8+ NKG2D+ T-cells has been found both necessary and sufficient to induce AA in $\mathrm{C} 3 \mathrm{H} / \mathrm{HeJ}$ mice. ${ }^{5,6}$ A predominant Th1 cytokine profile has been discovered at the site of AA lesions. ${ }^{3,7}$
Correspondence: Mariya Miteva Department of Dermatology and Cutaneous Surgery, University of Miami Miller School of Medicine, I600 NW I0th Avenue, Rosenstiel Medical Science Building, Room 2023A, 33136 Miami, FL, USA

Emailmmiteva@med.maimi.edu 
Recently, a genome-wide association study demonstrated a genetic predisposition to AA. ${ }^{7}$ Environmental insults, such as viral infections, trauma, or psychosocial stress, have also been suspected to possibly contribute to the development of the disease. ${ }^{3}$

Treatment options for AA have limited success, no cure has been found, and no therapy has been able to prevent disease relapse. ${ }^{1,2,8}$ Treatment options include topical, locally injected, or systemic steroids; topical immunotherapy; topical minoxidil; topical irritants such as anthralin; and systemic immunosuppressants such as cyclosporine or methotrexate. ${ }^{8,9}$ Success rates vary depending on the extent and duration of disease. Psychosocial support and therapy is also an important part of disease management, as this often disfiguring disease can be psychosocially burdensome.

The objectives of this review are to 1$)$ to assess the epidemiology of alopecia areata, including its incidence, prevalence, and distribution by sex and age - a brief description of the genome-wide association studies is also included; and 2) to assess its burden of disease including its comorbid psychiatric and medical conditions. Burden of disease is measured by disability-adjusted life years (DALYs), which combine years lost to disability (morbidity) and years lost to death (mortality) so that one DALY represents 1 year of healthy life lost. ${ }^{9}$

\section{Materials and methods}

A search of the literature was performed in order to answer the question: what is the epidemiology and burden of alopecia areata? A search was performed first on PubMed with the terms "alopecia areata epidemiology", which yielded 208 results. All titles and abstracts were scanned to determine whether each article answered the question. Further searches were performed including: "alopecia areata prevalence", "alopecia areata incidence", "alopecia areata distribution", "alopecia areata burden", "alopecia areata DALY", "alopecia areata demographics", "alopecia areata comorbidity", and "National Alopecia Areata Registry". Similar searches were also performed on Embase and Web of Science.
Articles chosen for inclusion were peer-reviewed with available full-text or abstract in English, providing primary data, and were published in the last 51 years. Additionally, secondary data generated by the World Health Organization (WHO) in the Global Burden of Disease Study were included. For the purpose of defining the epidemiology of AA, we selected articles with data on the prevalence, incidence, and distribution by sex or age. For the burden of disease, we selected articles with data on DALYs as well as associated psychiatric or medical comorbidities.

\section{Results \\ Epidemiology: prevalence and incidence}

Two population studies have measured the incidence and prevalence of AA, both based in Olmsted County, Minnesota, USA. ${ }^{10,11}$ Mirzoyev et al analyzed data from the Rochester Epidemiology Project, assessing 530 newly diagnosed patients with AA from 1990 to $2009 .{ }^{10}$ Estimated incidence was 20.9 per 100,000 person-years with a cumulative lifetime incidence of $2.1 \% .{ }^{10}$ The cumulative AA incidence increased almost linearly with age.$^{10}$ Almost 20 years prior, Safavi et al estimated the overall incidence of AA at 20.2 per 100,000 person-years with a lifetime incidence of $1.7 \%$ using data from 1975 through $1989 .{ }^{11}$ Three years prior to that, using data from a survey conducted between 1971 and 1974, prevalence was estimated at $0.1 \%$ to $0.2 \%$, with lifetime risk of $1.7 \% .^{12}$

Hospital-based studies from across the world have estimated the incidence of AA to be between $0.57 \%$ and $3.8 \%$, as detailed in Table $1 .{ }^{13-16}$ An estimated 2.4 million doctor office visits in the USA are for AA, which accounts for $25 \%$ of visits for all types of alopecia. ${ }^{17}$ Prevalence in Japan was calculated to be $2.45 \%{ }^{18}$ Episodes of AA last less than 6 months in the majority of patients..$^{13,19}$

\section{Epidemiology: family history of AA}

Patients with AA reporting a family history of the disease have been estimated between $0 \%$ and $8.6 \% .{ }^{13,16,20}$ In children, rates of family history of AA have been reported to be between

Table I Incidence of alopecia areata globally

\begin{tabular}{|c|c|c|c|c|c|}
\hline Incidence, \% & Number of persons in study & Country & Type of study & Comments & References \\
\hline 2.1 & 530 patients & USA & Population study & Lifetime incidence & 10 \\
\hline 1.7 & 292 patients & USA & Population study & Lifetime incidence & II \\
\hline 2 & $\mathrm{n} / \mathrm{a}$ & USA and Britain & Review and hospital-based study & & 14 \\
\hline 0.7 & 808 patients, 572 controls & India & Hospital-based study & & 15 \\
\hline 3.8 & 219 patients & Singapore & Hospital-based study & & 16 \\
\hline 0.57 & 187 patients & Mexico & Hospital-based study & & 13 \\
\hline
\end{tabular}

Abbreviation: n/a, not applicable. 
$10 \%$ and $51.6 \% .^{19,21-23}$ One study found that males were more likely to have a family history than females were. ${ }^{24}$

\section{Epidemiology: distribution by sex}

No significant difference in the incidence of AA was found between males and females in either of the two population studies. ${ }^{10,11}$ Ten different hospital-based studies from across the world, however, have cited a female predominance, ranging from a ratio of 2.6:1 to $1.2: 1 .^{13,15,16,22,24-29}$ Contrarily, four studies have shown a male predominance ranging from $2: 1$ to $1.1: 1 .^{15,20,30,31}$ In children, there was a male predominance at 1.4:1 in two studies, with one citing boys as having more severe involvement; a third study reported girls as having more severe disease. ${ }^{16,19,23}$

Male patients were reported as receiving a diagnosis of AA at an earlier age than female patients. ${ }^{24}$ Females were found to have a greater likelihood of extensive AA than males. ${ }^{19}$ Females were found to have higher rates of comorbid nail involvement and concomitant autoimmune disease, particularly thyroid disease. ${ }^{24}$ Some studies report no statistically significant differences in the age of onset, duration, or type of AA by sex or ethnicity. ${ }^{32}$

\section{Epidemiology: distribution by age and body site}

AA has historically been more prevalent in the younger age groups. ${ }^{33}$ The largest age group presenting for care was $21-40$-years of age, followed by the 1-20-year age group, the 41-60-year age group, and finally the 61-80-year age group. ${ }^{16}$ Similarly, peak in visits for AA has been reported in the $30-59$-year age group and the $31-35$-year age group. ${ }^{17,18}$

\section{Age of onset}

AA sufferers experience their first onset of AA by age 40 years in $82.6 \%-88 \%$ of patients and by age 20 years in $40.2 \%$ of patients..$^{15,16,20}$ The mean age of onset has been reported as between 25.2 and 36.3 years, as depicted in Table 2. ${ }^{10,16,20,32,34}$ Age of onset for females compared to males has been variously reported as lower (24.2 vs 26.7 years) ${ }^{16}$ the same, ${ }^{20}$ and higher (36.2 vs 31.5 years). ${ }^{10}$ In children, the mean age of onset has been reported as between ages 5 and 10 years. ${ }^{21-23,35}$

Table 2 Age of onset of alopecia areata globally

\begin{tabular}{llll}
\hline Age of onset, years & Country & Type of study & Reference \\
\hline 25.2 & Singapore & Hospital-based & 16 \\
28.98 & $\begin{array}{l}\text { People's Republic } \\
\text { of China }\end{array}$ & Hospital-based & 20 \\
36.3 & USA & Hospital-based & 32 \\
32.2 & Taiwan & Hospital-based & 34 \\
33.6 & USA & Population & 10 \\
\hline
\end{tabular}

\section{Epidemiology: distribution by body site}

The scalp is the most common site of involvement, with or without involvement of other body sites (such as the eyebrows, eyelashes, and beard). ${ }^{13,16}$ Specifically, the most common site was the occipital region, involved in $38.4 \%$ of males and $33.4 \%$ of females. ${ }^{36}$ Upon first presentation, $58 \%$ of adult patients had patchy hair loss with less than half the scalp involved. ${ }^{16}$ On the other hand, in children, 80\%-85\% had mild to moderate hair loss involving less than half the scalp. ${ }^{21-23,35}$ A later age of onset correlated with a less extensive alopecia, or in other words, onset in the first 2 decades was more often associated with severe alopecia. ${ }^{15,16,20}$ Alopecia totalis and universalis occurred in $7.3 \%$ of AA cases and always occurred before the age of 30 years. ${ }^{16}$

Nail changes occur in $10.5 \%-38 \%$ of AA patients, with common findings including pitting, trachyonychia, and longitudinal ridging. ${ }^{15,16,32}$ Nail changes correlated with disease severity, as they were found in more severe AA. ${ }^{15,16,32}$ Furthermore, nail dystrophy is a poor prognostic indictor of AA. ${ }^{16}$

\section{Results: burden of disease Burden of disease: DALYs}

In the Global Burden of Disease Study, WHO measured the global DALYs lost to AA in 2010 to be $1,332,800 .{ }^{37}$ For comparison, the DALYs of psoriasis are 1,050,660, and for diabetes mellitus they are 46,857,100. ${ }^{37}$ The DALYs for AA have been increasing linearly since 1990, when the global DALYs were under 1 million. No deaths have been reported from AA between 1990 and 2010. The disability weight for AA, which ranges from 0 to 1 , was $0.035 .{ }^{9} \mathrm{AA}$ is thought to account for $0.071 \%$ of total US DALYs. ${ }^{9}$

\section{Burden of disease: quality of life}

Over half of patients with AA experience poor health-related quality of life (QOL). ${ }^{38}$ Though patients of all ages and both sexes may experience decreased QOL with AA, risk factors for poor health-related QOL include age between 20 and 50 years, female sex, lightening of skin color, hair loss greater than $25 \%$, family stress, and job change. ${ }^{38}$ Patients with extensive AA experienced more adverse psychological effects than those with limited AA. ${ }^{16}$

\section{Burden of disease: psychiatric comorbidity}

A $66 \%-74 \%$ lifetime prevalence of psychiatric disorders has been reported in AA patients, with a 38\%-39\% lifetime 
prevalence of depression and a 39\%-62\% prevalence of generalized anxiety disorder. ${ }^{39-42}$ Adjustment disorders are also commonly found with AA. ${ }^{40}$ Table 3 outlines the rates of psychiatric disorders in AA patients compared to the general population. Approximately $50 \%$ of psychiatric disorders presented earlier than did AA, with the other half presenting after the onset of AA. ${ }^{42}$ Furthermore, stressful events preceding hair loss were recalled in $9.8 \%$ of adults and in $9.5 \%-80 \%$ of children. ${ }^{16,21,35}$ The rate of psychiatric comorbidity was influenced by the age of onset of AA, with an increased risk of depression in patients aged $<20$ years and an increased risk of anxiety and obsessive-compulsive disorder in those aged 40-59 years. ${ }^{42}$

No patients were acutely suicidal in a study of 45 AA patients, four with alopecia totalis and 28 with alopecia universalis. ${ }^{43}$ One patient in that study, however, answered yes to "dying is the best solution for me." Four patients affirmed that they "wish [they] were dead," and one denied that "life is still worth living." ${ }^{\prime 3}$ A recent letter to the editor, however, reports the death of four Australian males aged 14-17 years who were affected by AA with no known pre-existing psychological disorders, and with social withdrawal that began after the onset of the alopecia. ${ }^{44}$

\section{Burden of disease: comorbid medical conditions}

The incidence of atopy in patients with AA has been reported to be between $11 \%$ and $38.2 \% .{ }^{15,33,45,46}$ In children with AA, atopy is seen in $26.6 \%$ of patients, though one study found no significant personal history of atopy compared to controls. ${ }^{19,35}$ A personal or family history of atopy was reported in $60.7 \%$ of adults with AA and in $25 \%$ of children with AA. ${ }^{16,21}$ Specifically, atopic dermatitis was reported in $15.6 \%$ of adults with AA and in $39.5 \%$ of children with AA. ${ }^{13,47}$

Five percent of patients in one study were discovered to have subclinical hypothyroidism due to Hashimoto's thyroiditis. ${ }^{35}$ Seventy-five percent of those discovered to have Hashimoto's thyroiditis had a family history of thyroid disorder. ${ }^{35}$ Thyroid function abnormalities were found in

Table 3 Psychiatric disorders in alopecia areata (AA) patients compared to the general population

\begin{tabular}{lll}
\hline Disorder & AA patients, \% & $\begin{array}{l}\text { General } \\
\text { population, \% }\end{array}$ \\
\hline Major depression & 8.8 & $1.3-1.5$ \\
Generalized anxiety disorder & 18.2 & 2.5 \\
Social phobias & 3.5 & $0.9-2.2$ \\
Paranoid disorder & 4.4 & $<1$ \\
\hline
\end{tabular}

Notes: Data from Koo et al..$^{55}$
$8.9 \%$ of patients with AA. ${ }^{29}$ Similarly, the prevalence of thyroid peroxidase antibodies in AA patients was reported to be $17.7 \%$, about double that of the general population, with a female-to-male predominance of $6.7: 1 .{ }^{48}$ In general, AA patients were significantly more likely to have a family history of thyroid disorder. ${ }^{35}$

In addition to autoimmune thyroid disease, it has been long suspected that there is an association between AA and other autoimmune disorders such as vitiligo, systemic lupus erythematosus, psoriasis, inflammatory bowel disease, and rheumatoid arthritis. ${ }^{34,46}$ Incidence of the aforementioned disorders in AA can be seen in Table 4. Positive autoimmune antibodies (ANA, SMA, Anti-Tg, or PCA) were reported in $51.4 \%$ of patients with AA. ${ }^{29}$ Patients with a family history of vitiligo were more likely to develop extensive AA. ${ }^{15}$ Some studies, however, did not observe a correlation with autoimmune conditions in the AA patients or in family history of autoimmune disorders, with the exception of thyroid disorder. ${ }^{17,35}$

Most recently, diabetes mellitus was found in $11.1 \%$ of AA patients, as shown in Table $4 .{ }^{46}$ Significant increases in levels of insulin, c-peptide, and HOMA-IR were reported in AA patients compared to controls, suggesting increased insulin resistance in AA patients. ${ }^{49}$ Approximately $36.6 \%$ of AA patients in one study reported family history of diabetes mellitus type $2 .^{13}$

Down syndrome was found in $1.4 \%$ of AA patients with onset of disease before age 15 years and with extensive involvement in all of these patients. ${ }^{19}$

\section{Summary of genome-wide associations in AA}

A review on AA would not be complete without a mention of the current literature on genomic associations. Previously, genes in the human leukocyte antigen $(H L A)$ region were implicated in AA as well as some genes outside of HLA including PTPN22 and AIRE. ${ }^{50}$ In 2010, Petukhova et al published reports of a genome-wide association study that

Table 4 The incidence of autoimmune disorders in alopecia areata (AA)

\begin{tabular}{lll}
\hline Autoimmune disorder & Incidence in AA, \% & Reference \\
\hline Vitiligo & $1.8-7.0$ & $15,16,33,46,47$ \\
Thyroid disorder & $2.3-14.6$ & $16,33,34,46$ \\
Irritable bowel syndrome & 2.0 & 46 \\
Psoriasis \pm psoriatic arthritis & $1.9-6.3$ & 34,46 \\
Systemic lupus erythematosus & 1.5 & 34 \\
Rheumatoid arthritis & $0.9-3.9$ & $19,34,46$ \\
Diabetes mellitus & $0.4-11.1$ & 15,46 \\
\hline
\end{tabular}


discovered 139 single nucleotide polymorphisms (SNPs) associated with AA, mostly genes related to inflammation and immune-modulation, HLA, and cytokines. ${ }^{7}$ Specifically, those SNPs include: genes that control the activation and proliferation of regulatory T-cells; cytotoxic T lymphocyteassociated antigen 4; interleukins 2 and 21; interleukin-2 receptor A; EOS (or Ikaros family zinc finger 4); $H L A$; genes expressed within the hair follicle, including $P R D X 5$ and STX17; and the ULBP (cytomegalovirus UL16-binding protein) gene cluster on chromosome 6q25.1, encoding activating ligands of the natural killer cell receptor NKG2D., ${ }^{7,51}$ Using an Immunochip, Redler et al discovered that the strongest associations were observed for variants of the major histocompatibility complex class II DQ beta 1 ( $H L A-D Q B 1)$ and class II DQ alpha 2 (HLA-DQA2). ${ }^{51}$ Examples of SNPs associated with AA in genes that regulate T-cell function include the promoter regions of Foxp 3 and ICOSLG, and the CTLA4 CT60 polymorphism. ${ }^{52,53}$ Most recently, Betz et al discovered three novel loci: $A C O X L / B C L 2 L 11$ (BIM) (2q13); GARP (LRRC32) (11q13.5); and $S H 2 B 3(L N K) /$ ATXN2 (12q24.12). ${ }^{54}$

\section{Discussion and conclusion Epidemiology}

This systematic review provides a summary of the existing data on the worldwide incidence and prevalence of AA and its burden. Comparison between studies was attempted in relation to geography, age, and sex. Generally, a concern with the population studies is that their sample from a population register may not be representative of the true population at risk of AA. In fact, both population studies cited in the current review were based in Minnesota. ${ }^{10,11}$ However, there are also concerns that hospital-based studies may fail to provide an unbiased sample of the population at risk with respect to exposure status. These concerns aside, our literature search found that the estimated incidence of AA was approximately $2 \%$ in the population studies and close to $2 \%$ in hospitalbased studies globally (Table 1). ${ }^{10,11,13-16}$

There is no clear conclusion about whether the disease varies according to sex. There appears to be no significant difference in the incidence of AA between males and females as both formal population studies found none, and hospital-based studies are mixed in citing a female vs male predominance..$^{10,11,13,15,16,19,20,22-32}$ Again, hospital-based studies depend on access to care, and in some cases, willingness to participate in the study, which may bias the results.

Most patients experience their first onset of AA by age 40 years, with a peak of incidences occurring in their 20s and 30s (Table 2). ${ }^{10,15,16,20,32,34}$ The younger the age of onset, the greater the lifetime likelihood of extensive alopecia. ${ }^{15,16,20}$ Access to care and delay in presentation may also play a role in the age distribution seen. Family history of AA is present in a minority of patients with AA, and is seen more often in patients who present with AA as children. ${ }^{13,16,19-23,36}$

The scalp is involved with or without involvement of other body sites in almost all cases of AA, with the occipital scalp being the most commonly involved site. ${ }^{13,16,36}$ Nail involvement can be seen in severe AA and is a poor prognostic factor. ${ }^{15,16,32}$

\section{Burden}

The Global Burden of Disease Study, which estimates the DALYs for AA to be $1,332,800$, or a weighted 0.035 , may underestimate the true population-based prevalence and disease burden due to patients with AA who do not present for care. ${ }^{9}$ Furthermore, it considers the disability imposed by itch and disfigurement, but does not take into account emotional distress, interpersonal relationships, or financial impact. ${ }^{9}$

AA leads to decreased QOL in half of its sufferers and is associated with an approximately $70 \%$ lifetime prevalence of psychiatric disorders, most commonly depression, anxiety, and adjustment disorders. ${ }^{38-42}$ It has even been implicated in suicide. ${ }^{44}$ In about half of patients, however, psychiatric disorders presented earlier than AA, and in a portion of cases, stressful events were recalled preceding hair loss. ${ }^{16,21,35,42}$

AA appears to be associated with atopy and many autoimmune diseases including vitiligo and thyroid disorders. ${ }^{13,15,16,21,29,33-35,45-47,49}$ Some studies did not find association with autoimmune diseases. ${ }^{17,35}$ Genome-wide association studies, however, implicate genes in the HLA region and genes involved in inflammation and immune modulation in AA, many of which could plausibly affect multiple autoimmune conditions. ${ }^{7,51,54}$ Discrepancies in the incidence of associated conditions seen in Table 4 may, in part, be due to differences in the prevalence of atopy and autoimmune diseases across the globe and over time.

Extensive AA leads to greater disfigurement and psychosocial distress. Factors that increase the likelihood of extensive AA such as alopecia totalis and/or universalis include: younger age of onset; associated autoimmune or atopic disease, particularly thyroid disease and atopic dermatitis; nail changes; Down syndrome; positive family history of vitiligo; and positive family history of AA. ${ }^{15,16,32}$

In conclusion, studies on the occurrence of AA have contributed to a greater appreciation of the disease burden. 
The estimated high prevalence of $2 \%$ globally underscores the importance of disease awareness, of unveiling the role of genomics in influencing the likelihood of developing AA, and of discovering potential molecule-oriented treatments. Yet, epidemiological studies on AA are limited, and no clear conclusions can be drawn. Possible sources of heterogeneity in research results arise from 1) the different methodologies utilized to collect the data: hospital-based medical records, insurance databases, registries, or primary care databases, which may under-represent patients with no access to health care; and/or 2) case definition: self-diagnosis, primary care physician's diagnosis or dermatologist's diagnosis of AA. There is a need for international research collaborations using standardized methodology to address those knowledge gaps.

\section{Disclosure}

The authors report no conflicts of interest in this work.

\section{References}

1. Hordinsky MK. Overview of alopecia areata. J Investig Dermatol Symp Proc. 2013;16(1):S13-S15.

2. MacLean KJ, Tidman MJ. Alopecia areata: more than skin deep. Practitioner. 2013;257(1764):29-32,3.

3. Islam N, Leung PS, Huntley AC, Gershwin ME. The autoimmune basis of alopecia areata: a comprehensive review. Autoimmun Rev. 2015;14(2):81-89.

4. Christoph T, Muller-Rover S, Audring H, et al. The human hair follicle immune system: cellular composition and immune privilege. $\mathrm{Br} J$ Dermatol. 2000;142(5):862-873.

5. Cetin ED, Savk E, Uslu M, Eskin M, Karul A. Investigation of the inflammatory mechanisms in alopecia areata. Am J Dermatopathol. 2009;31(1):53-60.

6. Xing L, Dai Z, Jabbari A, et al. Alopecia areata is driven by cytotoxic T lymphocytes and is reversed by JAK inhibition. Nat Med. 2014; 20(9):1043-1049.

7. Petukhova L, Duvic M, Hordinsky M, et al. Genome-wide association study in alopecia areata implicates both innate and adaptive immunity. Nature. 2010;466(7302):113-117.

8. Shapiro J. Current treatment of alopecia areata. J Investig Dermatol Symp Proc. 2013;16(1):S42-S44.

9. Karimkhani C, Boyers LN, Prescott L, et al. Global burden of skin disease as reflected in Cochrane Database of Systematic Reviews. JAMA Dermatol. 2014;150(9):945-951.

10. Mirzoyev SA, Schrum AG, Davis MD, Torgerson RR. Lifetime incidence risk of alopecia areata estimated at $2.1 \%$ by Rochester Epidemiology Project, 1990-2009. J Invest Dermatol. 2014;134(4): 1141-1142.

11. Safavi KH, Muller SA, Suman VJ, Moshell AN, Melton LJ 3rd. Incidence of alopecia areata in Olmsted County, Minnesota, 1975 through 1989. Mayo Clin Proc. 1995;70(7):628-633.

12. Safavi K. Prevalence of alopecia areata in the First National Health and Nutrition Examination Survey. Arch Dermatol. 1992;128(5):702.

13. Guzmán-Sánchez DA, Villanueva-Quintero GD, Alfaro Alfaro N, McMichael A. A clinical study of alopecia areata in Mexico. Int $J$ Dermatol. 2007;46(12):1308-1310.

14. Price VH. Alopecia areata: clinical aspects. J Invest Dermatol. 1991; 96(5):68S.

15. Sharma VK, Dawn G, Kumar B. Profile of alopecia areata in Northern India. Int J Dermatol. 1996;35(1):22-27.
16. Tan E, Tay YK, Goh CL, Chin Giam Y. The pattern and profile of alopecia areata in Singapore - a study of 219 Asians. Int J Dermatol. 2002;41(11):748-753.

17. McMichael AJ, Pearce DJ, Wasserman D, et al. Alopecia in the United States: outpatient utilization and common prescribing patterns. $J \mathrm{Am}$ Acad Dermatol. 2007;57(2 Suppl):S49-S51.

18. Furue M, Yamazaki S, Jimbow K, et al. Prevalence of dermatological disorders in Japan: a nationwide, cross-sectional, seasonal, multicenter, hospital-based study. J Dermatol. 2011;38(4):310-320.

19. Tan E, Tay YK, Giam YC. A clinical study of childhood alopecia areata in Singapore. Pediatr Dermatol. 2002;19(4):298-301.

20. Yang S, Yang J, Liu JB, et al. The genetic epidemiology of alopecia areata in China. Br J Dermatol. 2004;151(1):16-23.

21. Rocha J, Ventura F, Vieira AP, Pinheiro AR, Fernandes S, Brito C. [Alopecia areata: a retrospective study of the paediatric dermatology department (2000-2008)]. Acta Med Port. 2011;24(2):207-214. Portuguese.

22. Nanda A, Al-Fouzan AS, Al-Hasawi F. Alopecia areata in children: a clinical profile. Pediatr Dermatol. 2002;19(6):482-485.

23. Xiao FL, Yang S, Liu JB, et al. The epidemiology of childhood alopecia areata in China: a study of 226 patients. Pediatr Dermatol. 2006;23(1): 13-18.

24. Lundin M, Chawa S, Sachdev A, Bhanusali D, Seiffert-Sinha K, Sinha AA. Gender differences in alopecia areata. J Drugs Dermatol. 2014;13(4):409-413.

25. Barahmani N, Schabath MB, Duvic M; National Alopecia Areata Registry. History of atopy or autoimmunity increases risk of alopecia areata. J Am Acad Dermatol. 2009;61(4):581-591.

26. Shellow WV, Edwards JE, Koo JY. Profile of alopecia areata: a questionnaire analysis of patient and family. Int J Dermatol. 1992; 31(3):186-189.

27. Blaumeiser B, van der Goot I, Fimmers R, et al. Familial aggregation of alopecia areata. J Am Acad Dermatol. 2006;54(4):627-632.

28. Kyriakis KP, Paltatzidou K, Kosma E, Sofouri E, Tadros A, Rachioti E. Alopecia areata prevalence by gender and age. J Eur Acad Dermatol Venereol. 2009;23(5):572-573.

29. Seyrafi H, Akhiani M, Abbasi H, Mirpour S, Gholamrezanezhad A. Evaluation of the profile of alopecia areata and the prevalence of thyroid function test abnormalities and serum autoantibodies in Iranian patients. BMC Dermatol. 2005;5:11.

30. Kavak A, Yeşildal N, Parlak AH, et al. Alopecia areata in Turkey: demographic and clinical features. J Eur Acad Dermatol Venereol. 2008;22(8):977-981.

31. Jain S, Marfatia YS. Alopecia areata - pattern in industrial city of Baroda. Indian J Dermatol Venereol Leprol. 2003;69(2):81-82.

32. Goh C, Finkel M, Christos PJ, Sinha AA. Profile of 513 patients with alopecia areata: associations of disease subtypes with atopy, autoimmune disease and positive family history. J Eur Acad Dermatol Venereol. 2006;20(9):1055-1060.

33. Muller SA, Winkelmann RK. Alopecia Areata. An evaluation of 736 patients. Arch Dermatol. 1963;88:290-297.

34. Chu SY, Chen YJ, Tseng WC, et al. Comorbidity profiles among patients with alopecia areata: the importance of onset age, a nationwide population-based study. J Am Acad Dermatol. 2011;65(5): 949-956.

35. Kakourou T, Karachristou K, Chrousos G. A case series of alopecia areata in children: impact of personal and family history of stress and autoimmunity. J Eur Acad Dermatol Venereol. 2007;21(3): 356-359.

36. Tak WJ, Chung YS, Ro BI. [A clinical study on alopecia areata (1996-2000) (TGF-VI)]. Korean J Dermatol. 2002;40(7):791-800. Korean.

37. Search GBD Data [webpage on the Internet]. Seattle, WA: Institute for Health Metrics and Evaluation; 2014 [cited February 13, 2015]. Available from: http://www.healthdata.org/search-gbd-data?s=Alopecia $\% 20$ areata. Accessed February 13, 2015. 
38. Shi Q, Duvic M, Osei JS, et al. Health-Related Quality of Life (HRQoL) in alopecia areata patients - a secondary analysis of the National Alopecia Areata Registry Data. J Investig Dermatol Symp Proc. 2013;16(1):S49-S50.

39. Colón EA, Popkin MK, Callies AL, Dessert NJ, Hordinsky MK. Lifetime prevalence of psychiatric disorders in patients with alopecia areata. Compr Psychiatry. 1991;32(3):245-251.

40. Ruiz-Doblado S, Carrizosa A, García-Hernández MJ. Alopecia areata: psychiatric comorbidity and adjustment to illness. Int J Dermatol. 2003;42(6):434-437.

41. Sellami R, Masmoudi J, Ouali U, et al. The relationship between alopecia areata and alexithymia, anxiety and depression: a case-control study. Indian J Dermatol. 2014;59(4):421.

42. Chu SY, Chen YJ, Tseng WC, et al. Psychiatric comorbidities in patients with alopecia areata in Taiwan: a case-control study. Br J Dermatol. 2012;166(3):525-531.

43. Gupta MA, Gupta AK. Depression and suicidal ideation in dermatology patients with acne, alopecia areata, atopic dermatitis and psoriasis Br J Dermatol. 1998;139(5):846-850.

44. Sinclair RD. Alopecia areata and suicide of children. Med J Aust. 2014;200(3):145.

45. Tosti A. Practice gaps. Alopecia areata and comorbid conditions. JAMA Dermatol. 2013;149(7):794.

46. Huang KP, Mullangi S, Guo Y, Qureshi AA. Autoimmune, atopic, and mental health comorbid conditions associated with alopecia areata in the United States. JAMA Dermatol. 2013;149(7):789-794.

47. Nnoruka EN, Obiagboso I, Maduechesi C. Hair loss in children in South-East Nigeria: common and uncommon cases. Int J Dermatol. 2007;46 Suppl 1:18-22.
48. Baars MP, Greebe RJ, Pop VJ. High prevalence of thyroid peroxidase antibodies in patients with alopecia areata. J Eur Acad Dermatol Venereol. 2013;27(1):e137-e139.

49. Karadag AS, Ertugrul DT, Bilgili SG, Takci Z, Tutal E, Yilmaz H. Insulin resistance is increased in alopecia areata patients. Cutan Ocul Toxicol. 2013;32(2):102-106.

50. Gilhar A, Paus R, Kalish RS. Lymphocytes, neuropeptides, and genes involved in alopecia areata. J Clin Invest. 2007;117(8):2019-2027.

51. Redler S, Angisch M, Heilmann S, et al. Immunochip-based analysis: high-density genotyping of immune-related Loci sheds further light on the autoimmune genetic architecture of alopecia areata. J Invest Dermatol. 2015;135(3):919-921.

52. Conteduca G, Rossi A, Megiorni F, et al. Single nucleotide polymorphisms in the promoter regions of Foxp3 and ICOSLG genes are associated with alopecia areata. Clin Exp Med. 2014;14(1):91-97.

53. Megiorni F, Mora B, Maxia C, Gerardi M, Pizzuti A, Rossi A. Cytotoxic T-lymphocyte antigen 4 (CTLA4) +49AG and CT60 gene polymorphisms in alopecia areata: a case-control association study in the Italian population. Arch Dermatol Res. 2013;305(7):665-670.

54. Betz RC, Petukhova L, Ripke S, et al. Genome-wide meta-analysis in alopecia areata resolves HLA associations and reveals two new susceptibility loci. Nat Commun. 2015;6:5966.

55. Koo JY, Shellow WV, Hallman CP, Edwards JE. Alopecia areata and increased prevalence of psychiatric disorders. Int J Dermatol. 1994;33(12):849-850.
Clinical, Cosmetic and Investigational Dermatology

\section{Publish your work in this journal}

Clinical, Cosmetic and Investigational Dermatology is an international, peer-reviewed, open access, online journal that focuses on the latest clinical and experimental research in all aspects of skin disease and cosmetic interventions. All areas of dermatology will be covered; contributions will be welcomed from all clinicians and

\section{Dovepress}

basic science researchers globally. This journal is indexed on CAS The manuscript management system is completely online and includes a very quick and fair peer-review system, which is all easy to use. Visit http://www.dovepress.com/testimonials.php to read real quotes from published authors. 